\title{
Efeito da duração do tratamento com progestágeno e da maturidade sexual na taxa de prenhez em novilhas de corte: avaliação econômica e biológica
}

\section{Luiz Francisco Machado Pfeifer ${ }^{1}$, Eduardo Madeira Castilho ${ }^{2}$, Victor Fernando Büttow Roll ${ }^{1}$, Augusto Schneider ${ }^{3}$, Evâneo Alcides Ziguer ${ }^{1}$, Nelson José Laurino Dionello ${ }^{1}$}

\footnotetext{
${ }^{1}$ Departamento de Zootecnia, Universidade Federal de Pelotas.

2 Médico Veterinário.

${ }^{3}$ Centro de Biotecnologia, Universidade Federal de Pelotas.
}

RESUMO - Os objetivos neste experimento foram avaliar o efeito do tempo de exposição a um progestágeno (P4) na taxa de prenhez de novilhas púberes e pré-púberes inseminadas em tempo-fixo (IATF) e estudar o retorno econômico dos protocolos utilizados, por meio da comparação desses protocolos ao sistema tradicional de acasalamento natural aos 26 meses de idade. Foram utilizadas 114 novilhas da raça Bradford com 16 meses de idade, classificadas em quatro grupos de acordo com o tratamento e a maturidade sexual: GPPLP - pré-puberes, longo tratamento com P4 ( $\mathrm{n}=21$ ); e GPPCP pré-puberes, curto tratamento com $(\mathrm{n}=19)$; GPLP - púberes, longo tratamento com P4 (n=34); e GPCP - púberes, curto tratamento com P4 $(n=40)$. As novilhas do GPPLP e GPLP receberam no dia -8 um pessário intravaginal impregnado com acetato de medroxiprogesterona. No dia 0 , o pessário foi removido e outro foi inserido, juntamente com uma injeção de $2,5 \mathrm{mg}$ de benzoato de estradiol (BE). No dia 8, o pessário foi retirado e as novilhas receberam $0,5 \mathrm{mg}$ de $\mathrm{BE}$ e $250 \mu \mathrm{g}$ de cloprostenol sódico. A inseminação artificial em tempo fixo (IATF) foi realizada 54 horas depois. As novilhas do GPPCP e GPCP receberam o mesmo tratamento de sincronização dos grupos GPPLP e GPLP, com exceção do AMP do dia -8 ao dia 0. A taxa de prenhez foi maior nas novilhas púberes $(60,3 \%)$ que nas pré-puberes $(35,0 \%)$. No entanto, não foi detectado efeito do tempo de tratamento na taxa de prenhez nas novilhas púberes e pré-puberes. A resposta econômica na utilização de protocolos com P4 foi maior em novilhas aos 16 meses que no sistema de acasalamento aos 26 meses. A exposição prolongada a um P4 não foi eficiente para aumentar a taxa de prenhez de novilhas submetidas a IATF.

Palavras-chave: bovinos, progesterona, puberdade, rentabilidade, sincronização de ovulação

\section{Effect of the duration of progestagen exposure and sexual maturity on the pregnancy rate of beef heifers: economic and biological evaluation}

\begin{abstract}
The aim of this experiment was to evaluate the effect of the duration of progestagen (P4) exposure and sexual maturity of prepubertal and pubertal heifers submitted to timed artificial insemination (TAI). A hundred and fourteen 16- month old Bradford heifers were classified, according treatment and sexual maturity, in 4 groups: PPLPG (prepubertal long exposure to P4, n=21), PPSPG (prepubertal short exposure to P4, $n=19$ ), PLPG (pubertal long exposure to $\mathrm{P} 4, \mathrm{n}=34$ ) and PSPG (pubertal short exposure to $\mathrm{P} 4, \mathrm{n}=40$ ). On day -8 , the heifers from PPLPG (prepubertal long exposure to progestagen) and PLPG (pubertal long exposure to progestagen) received an intravaginal sponge impregnated with medroxiprogesterone acetate (MAP). On day 0 the sponge was removed and a new sponge was inserted, together with an injection of $2.5 \mathrm{mg}$ estradiol benzoate (EB). On day 8 the sponge was removed and the heifers received an injection of $0.5 \mathrm{mg} \mathrm{EB}$ and $250 \mathrm{ig}$ sodium cloprostenol. The TAI was performed 54 hours after sponge withdrawal. The heifers from PPSPG and PSPG received the same synchronization treatment, except for the additional MAP from day -8 to day 0 . The pregnancy rate was higher in pubertal (60.3\%) than in pre-pubertal $(35.0 \%)$ heifers. However, there was no effect of the duration of treatment in the pregnancy rate between pubertal and prepubertal heifers. The second part of this study aimed to evaluate the economical return of the protocols and compare them with the traditional breeding system at 26 months of age. A better economic response with the use of protocols associating P4 in heifers at 16 months was observed compared to the traditional system at 26 months of age. The prolonged exposure to $\mathrm{P} 4$ did not improved pregnancy rate in heifers submitted to TAI.
\end{abstract}

Key Words: cattle, economical return, ovulation synchronization, progesterone, puberty 


\section{Introdução}

A produtividade do rebanho aumenta quando novilhas parem precocemente em sua primeira estação de parição (Lesmeister et al., 1973). Para que isso ocorra, é necessário que as novilhas concebam o mais cedo possível dentro da primeira estação de acasalamento para parirem no início da temporada subsequente, o que aumenta a possibilidade de tornarem-se gestantes novamente (Lobato \& Azambuja, 2002).

Vários estudos foram conduzidos com o uso de progestágenos no intuito de esclarecer a fisiologia e intervir no processo de aquisição da maturidade sexual (Tanaka et al., 1995; Hall et al., 1997; Rasby et al., 1998; Lucy et al., 2001; Gregory \& Rocha, 2004). Aparentemente, para que uma novilha apresente um corpo lúteo funcional seguido de fase luteal de duração normal, ocorrem duas elevações transientes de progesterona (Donaldson et al., 1970; Gonzalez-Padilla et al., 1975a; Gonzalez-Padilla et al., 1975b). A progesterona pode antecipar a puberdade, uma vez que reduz o feedback negativo do estradiol no hipotálamo, pela redução do número de receptores para estradiol (Day et al., 1984) confirmam a necessidade de exposição do trato reprodutivo à progesterona para que os primeiros ciclos estrais férteis possam ocorrer.

Neste contexto, as alternativas hormonais que induzem a ovulação pelo uso de progesterona (Tauck et al., 2007; Imwalle et al., 1998; Anderson et al., 1996) podem ser viáveis para antecipar a puberdade em novilhas. No entanto, quando são utilizadas inadequadamente, podem comprometer os custos de produção, em virtude dos resultados econômicos insatisfatórios, que inviabilizam o uso dessas alternativas. Dessa forma, a análise de custos permite verificar como as alternativas hormonais empregadas em um processo de produção estão sendo remuneradas, além de possibilitar a avaliação da rentabilidade em comparação a outras alternativas de emprego do tempo e capital. Por meio da análise dos custos incorridos na produção de carne, o produtor pode então fixar diretrizes e corrigir distorções oriundos do mau emprego dessas técnicas para possibilitar a sobrevivência do sistema de produção (Lopes \& Carvalho, 2003).

O objetivo neste estudo foi avaliar o efeito da duração do tratamento com progestágeno na taxa de prenhez de novilhas púberes e pré-púberes inseminadas artificialmente em tempo-fixo (IATF), bem como o retorno econômico dos protocolos utilizados em comparação a um sistema simulado de acasalamento natural aos 26 meses de idade.

\section{Material e Métodos}

Foram utilizadas neste estudo 114 novilhas da raça Bradford com 13 a 16 meses de idade, submetidas a um sistema de pastejo em campo nativo com lotação de 0,43 unidade animal por hectare (UA/ha). Durante o inverno, os animais foram alimentados em pastagem cultivada de aveia e azevém, atingindo no início do período experimental (dezembro de 2006) peso médio de $295 \mathrm{~kg}$.

Antes do início do experimento, as novilhas foram submetidas a dois exames ginecológicos, com intervalo de 10 dias, para avaliação do tônus uterino e da presença de corpo lúteo no ovário, e classificadas em dois grupos: púberes $(n=74)$ e pré-puberes $(n=40)$. Em seguida, foram divididas aleatoriamente, de acordo com a maturidade sexual, em quatro grupos experimentais: GPLP - grupo púbere tratamento longo com progestágeno $(n=34)$; grupo púbere tratamento curto com progestágeno ( $\mathrm{GPCP}, \mathrm{n}=40$ ), grupo pré-púbere tratamento longo com progestágeno (GPPLP, $n=21$ ) e grupo pré-púbere tratamento curto com progestágeno (GPPCP, $n=19$ ) (Figura 1).

As novilhas do GPLP e GPPLP receberam no dia -8 um dispositivo intravaginal impregnado com $250 \mathrm{mg}$ de acetato de medroxiprogesterona (AMP) durante 8 dias. Após a remoção do dispositivo (dia 0 ), as novilhas receberam um novo dispositivo intravaginal impregnado com $250 \mathrm{mg}$ de AMP e aplicação intramuscular de $2,5 \mathrm{mg}$ de benzoato de estradiol (BE). O dispositivo foi removido no dia 8, juntamente com a aplicação de $250 \mu \mathrm{g}$ de cloprostenol sódico e $0,5 \mathrm{mg}$ de benzoato de estradiol. Após 54 horas da retirada dos pessários, foi realizada a inseminação artificial em tempo fixo. As novilhas do GPCP e do GPPCP, no entanto, foram submetidas ao mesmo protocolo de sincronização, com exceção da pré-exposição ao AMP do dia -8 ao dia 0 (Figura 1 ).

O diagnóstico de gestação foi realizado por ultrasonografia (PIEMEDICAL ANSWER, equipado com sonda convexa de $5 \mathrm{Mhz}$ ) 30 dias após a inseminação artificial em tempo fixo.

Os dados biológicos e econômicos foram tabulados no programa Microsoft Excel ${ }^{\circledR}$ para as simulações econômicas. $\mathrm{Na}$ avaliação econômica, utilizaram-se os dados biológicos obtidos (taxa de prenhez), porém, para trabalhar com um valor econômico mais expressivo, realizou-se simulação de gastos e receitas para 100 fêmeas em cada grupo experimental.

Para comparação dos resultados econômicos, foram feitas projeções de desempenho e custo do acasalamento das novilhas e vacas primíparas aos 26 meses de idade em 

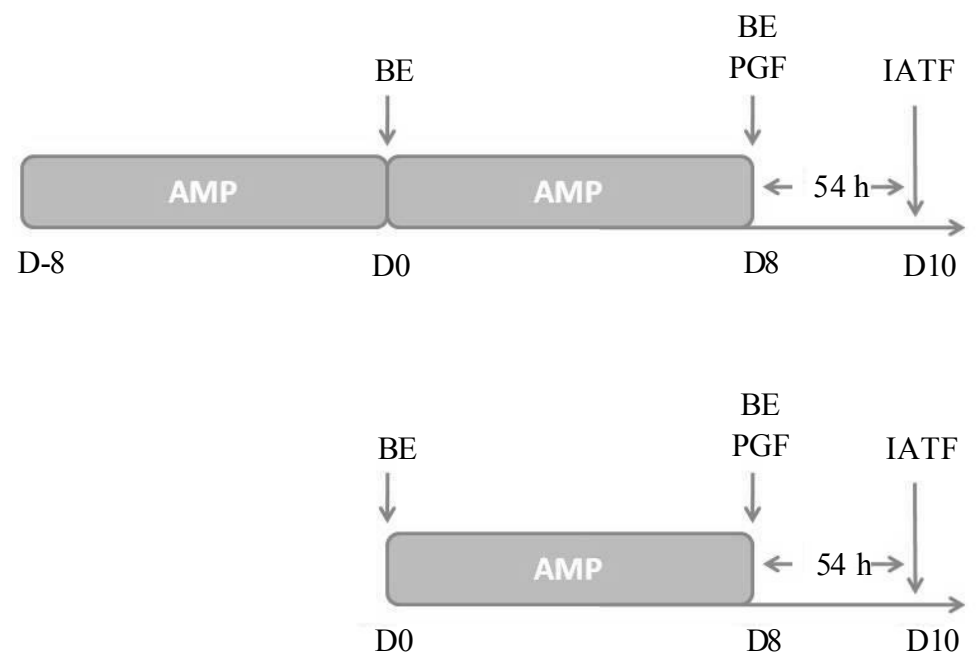

Figura 1 - Protocolo utilizado para a sincronização de ovulação nos grupos experimentais.

1 - acetato de medroxiprogesterona (250 mg); 2 - benzoato de estradiol (2,5 mg); 3 - benzoato de estradiol (0,5 mg); 4 - análogo da prostaglandina $(250 \mu \mathrm{g})$.

sistema de monta natural. Para isso, os grupos experimentais foram comparados a um grupo controle fictício (GCF, $\mathrm{n}=100$ ), que representa o sistema de acasalamento de novilhas somente aos 26 meses de idade, tradicionalmente usado (Silva et al., 2005), considerando taxa de prenhez de $90 \%$ (Vieira et al., 2006). Também foram feitas projeções para as vacas primíparas dos grupos experimentais que foram submetidas a inseminação artificial em tempo fixo aos 16 meses, considerando taxa de prenhez de $90 \%$ para aquelas que não conceberam (Vieira et al., 2006) e de $80 \%$ para aquelas que conceberam na inseminação artificial em tempo fixo (Lobato et al., 1998b). O período de monta simulado foi de 100 dias(Lobato et al., 1998a).

As projeções de custos e receitas iniciaram a partir da sincronização das novilhas aos 16 meses de idade e prosseguiram até a venda dos bezerros concebidos no acasalamento aos 26 meses de idade. $O$ custo de manutenção das vacas em um ciclo produtivo foi ajustado de acordo com dados de Castilho \& Pfeifer (2005), considerando desembolso anual de $\mathrm{R} \$ 123,64$, relacionado ao custo de oportunidade da terra, aos gastos com nutrição e sanidade e às despesas parciais com funcionários (relação de tempo destinado somente às fêmeas durante as distintas fases do ciclo de produção). Foi considerado período de manutenção de três anos, necessário para a venda dos bezerros concebidos no acasalamento aos 26 meses.

O custo de implantação da técnica de sincronização de cios e inseminação artificial em tempo fixo em cada grupo foi determinado considerando os gastos com hormônios, deslocamento da equipe, valor das doses de sêmen, gastos com inseminador e diagnóstico de gestação. Para contabilização das despesas referentes ao segundo período de acasalamento (aos 26 meses) do GPLP, GPCP, GPPLP, GPPCP e GCF, foram considerados fatores como vida útil média dos reprodutores, número de fêmeas cobertas por um touro durante a estação reprodutiva, custo médio do reprodutor, valor residual do macho (valor pago pelo frigorífico ao ser vendido para o abate), custo de oportunidade da área destinada ao reprodutor e despesas com nutrição e sanidade.

O cálculo do custo de manutenção dos bezerros concebidos no acasalamento aos 26 meses foi realizado considerando os desembolsos com esta categoria de animais do nascimento à desmama (210 dias). Os gastos considerados foram: vacinação, evermifugação, custo de oportunidade da área ocupada pelos animais e gastos com funcionários (proporcional ao tempo destinado aos bezerros).

A simulação econômica também incluiu o fornecimento de alimento energético para as novilhas acasaladas aos 16 meses no período pós-parto e uso de creep-feeding para os bezerros. Para isso, foi necessário calcular a depreciação da estrutura de cocho para os bezerros e as vacas de cada grupo. Considerando que o número de novilhas e bezerros a serem suplementados nos grupos GPLP, GPCP, GPPLP, GPPCP eram similares, foram considerados valores de $\mathrm{R} \$ 350,00$ e R \$175,00 para os lotes púberes e pré-puberes, respectivamente.

No cálculo do custo de suplementação pós-parto das novilhas durante 120 dias, foram considerados peso médio para as novilhas de $360 \mathrm{~kg}$ (Lobato et al., 1998), consumo médio diário de $0,5 \%$ de seu peso vivo e custo de 


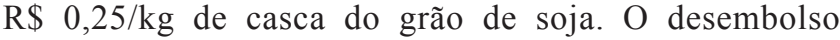
considerado foi de R \$55,50/animal, enquanto, no cálculo do custo de suplementação dos bezerros do desmame à venda (210 dias), foi considerada ração consumida em um período de 180 dias, com consumo médio de $1 \mathrm{~kg} / \mathrm{dia} /$ bezerro. O custo da ração pós-desmama foi de $\mathrm{R} \$ 0,54 / \mathrm{kg}$, resultando em custo de $\mathrm{R} \$ 97,20 /$ bezerro nesse período.

Para cálculo da receita da venda de bezerros obtidos com a inseminação artificial em tempo fixo aos 16 meses, foi considerada a venda total dos bezerros aos 210 dias de idade. Estimaram-se um peso médio ao nascimento de $34 \mathrm{~kg}$ e taxa de ganho médio diário de $0,67 \mathrm{~kg}$ (Lobato et al., 1998a), totalizando $174 \mathrm{~kg}$ aos 210 dias de idade. Considerou-se o preço médio do $\mathrm{kg}$ para esta categoria de $\mathrm{R} \$ 2,60$.

Os dados referentes à taxa de prenhez foram avaliados pelo teste do qui-quadrado pelo programa Statistix ${ }^{\circledR}$ (2004). A rentabilidade das alternativas e dos sistemas utilizados no trabalho foi expressa em valor presente líquido (VPL), taxa interna de retorno (TIR) e taxa mínima de atratividade (TMA) (Buarque, 1984).

\section{Resultados e Discussão}

A taxa de prenhez foi maior $(\mathrm{P}<0,05)$ entre novilhas púberes (GPLP e GPCP combinados), 60,3\% (44/74), que entre novilhas pré-puberes (GPPLP e GPPCP combinados), $35,0 \%$ (14/40). No entanto, não houve efeito da duração do tratamento com progestágeno e da maturidade sexual, pois não foi encontrada diferença $(\mathrm{P}>0,05)$ na taxa de prenhez entre as fêmeas pré-púberes submetidas e aquelas não submetidas à pré-exposição ao progestágeno, de 33\% (7/21) e 37\% (7/19) para os grupos GPPLP e GPPCP, respectivamente. Não houve diferença $(\mathrm{P}>0,05)$ na taxa de prenhez entre o $\operatorname{GPLP}(65 \%, 22 / 34)$ e o $\operatorname{GPCP}(58 \%, 23 / 40)$. Quando avaliada a taxa de prenhez sem considerar a maturidade sexual das novilhas, foi registrada taxa de 51,8\% (28/54) para o GPLP e GPPLP combinados e de 50,8\% (30/59) para o GPCP e GPPCP, mas não foi observada diferença $(\mathrm{P}>0,05)$.

Os resultados deste experimento diferem do relatado por Tauck et al. (2007) de que a exposição a progesterona por 14 dias pode aumentar a taxa de prenhez em comparação à exposição por 7 dias em novilhas de corte (Lamb et al., 2006), o que indica que o aumento no tempo de exposição à progesterona pode aumentar a taxa de concepção.

A exposição a um progestágeno por 10 dias é eficiente para induzir a puberdade apenas em novilhas mais velhas, independentemente do padrão anterior de ganho de peso (Hall et al., 1997). A eficácia dos tratamentos com progestágenos para induzir a puberdade depende de vários fatores, incluindo idade, raça, peso e grau de desenvolvimento folicular das novilhas antes do tratamento (Gonzalez-Padilla et al., 1975c). Neste experimento, apesar de as novilhas apresentarem peso corporal ideal de $295 \mathrm{~kg}$ (Gregory \& Rocha, 2004), algumas podiam ainda não ter idade suficiente para que a puberdade fosse induzida. A exposição ao progestágeno induz aumento da frequência da pulsatilidade de hormônio luteinizante nas novilhas de maneira similar ao observado no processo espontâneo de evolução para a puberdade (Day et al., 1984). De acordo com Anderson et al. (1996), a habilidade do progestágeno em induzir a puberdade está relacionada à maturidade do sistema neuroendócrino, o qual regula a secreção de hormônio luteinizante. Essa hipótese está baseada no fato de a progesterona causar redução no número de receptores de estradiol no sistema neural, o que modularia a ação do feedback negativo do estradiol nos neurônios liberadores de GnRH (Day et al., 1984).

É possível que não seja necessária exposição prolongada ao progestágeno para que a puberdade seja induzida, visto que o tratamento com progesterona por 7 dias é capaz de induzir a ovulação seguida da formação de um corpo lúteo de duração normal em novilhas (Rasby et al., 1998). Isso pode explicar porque não foi encontrada diferença entre as novilhas que receberam tratamento longo e curto com progestágeno, pois a exposição ao progestágeno por 8 dias teria sido suficiente para induzir a puberdade, o que dispensa tratamento prolongado.

O custo de implantação da técnica de sincronização de cios foi de $\mathrm{R} \$ 3.000,00, \mathrm{R} \$ 2.500,00, \mathrm{R} \$ 3.000,00 \mathrm{e} \mathrm{R} \$ 2.500,00$ para o GPLP, GPCP, GPPLP e GPPCP, respectivamente (Tabela 1). No entanto, para o acasalamento de cada um dos cinco grupos aos 26 meses de idade, foi considerado desembolso de R $\$ 1.000,00$, pois, segundo Castilho \& Pfeifer (2005), para acasalar um grupo de 400 vacas, é necessário desembolso anual de $\mathrm{R} \$ 4.000,00$ para despesas com monta natural.

O custo de suplementação pós-parto por um período de 120 dias das novilhas que haviam sido acasaladas aos 16 meses foi multiplicado pelo número de fêmeas a receberem suplemento em cada grupo, chegando aos respectivos valores: $\mathrm{R} \$ 3.607,50(65 \times \mathrm{R} \$ 55,50), \mathrm{R} \$ 3.219,00(58 \mathrm{x}$ $\mathrm{R} \$ 55,50) ; \mathrm{R} \$ 1.831,50(33 \times \mathrm{R} \$ 55,50)$ e $\mathrm{R} \$ 2.053,50(37 \mathrm{x}$ $\mathrm{R} \$ 55,50)$ para o GPLP, GPCP, GPPLP e GPPCP, respectivamente. No cálculo do custo de suplementação dos bezerros do desmame à venda, obtiveram-se os seguintes gastos $\mathrm{R} \$ 6.318,00$ (65 x R \$ 97,20), R \$ 5.637,60 (58 x R \$ 97,20), R\$3.207,60 (33 x R \$ 97,20) e R \$3.596,40 (37 x R\$ 97,20) para os grupos GPLP, GPCP, GPPLP e GPPCP, respectivamente. 
Tabela 1 - Demonstração dos custos totais nos grupos simulados, em R $\$$, e dos custos incidentes sobre os cinco grupos, em R $\$$

\begin{tabular}{|c|c|c|c|c|c|}
\hline Variável & GPLP & GPCP & GPPLP & GPPCP & GCF \\
\hline Custo de manutenção de vacas, referente a 3 anos & $37.092,00$ & $37.092,00$ & $37.092,00$ & $37.092,00$ & $37.092,00$ \\
\hline Custo da monta natural aos 26 meses & $1.000,00$ & $1.000,00$ & $1.000,00$ & $1.000,00$ & $1.000,00$ \\
\hline $\begin{array}{l}\text { Custo de manutenção dos bezerros - acasalamento } \\
\text { aos } 26 \text { meses }\end{array}$ & $2.279,55$ & $2.298,66$ & $2.366,91$ & $2.355,99$ & $2.457,00$ \\
\hline
\end{tabular}

Custos incidentes sobre os grupos que foram

acasalados aos 16 meses, em $\mathrm{R} \$$

\begin{tabular}{|c|c|c|c|c|c|}
\hline $\begin{array}{l}\text { Custo de implantação da técnica de sincronização } \\
\text { de cio e IATF }\end{array}$ & $3.000,00$ & $2.500,00$ & $3.000,00$ & $2.500,00$ & - \\
\hline Custos de depreciação de cochos & 350,00 & 350,00 & 175,00 & 175,00 & - \\
\hline Custo de suplementação pós-parto das novilhas & $3.607,50$ & $3.219,00$ & $1.831,50$ & $2.053,50$ & - \\
\hline Custo de suplementação dos bezerros & $6.318,00$ & $5.637,60$ & $3.207,60$ & $3.596,40$ & - \\
\hline Custo total & $53.974,05$ & $52.411,66$ & $48.942,41$ & $49.049,49$ & $40.759,00$ \\
\hline
\end{tabular}

O gasto diário por bezerro, concebido no acasalamento aos 26 meses, do nascimento à desmama foi de R $\$ 0,13 \mathrm{e}$, considerando que o desmame foi realizado aos 210 dias, o custo de manutenção foi de $\mathrm{R} \$ 27,30 /$ bezerro (Castilho \& Pfeifer, 2005). Portanto, os custos de manutenção dos bezerros concebidos no acasalamento aos 26 meses foram os seguintes: GPLP R \$2.279,55; GPCP R \$2.298,66; GPPLP $\mathrm{R} \$ 2.366,91 ;$ GPPCP R $\$ 2.355,99$ e GCF R $\$ 2.457,00$.

A receita com a venda dos bezerros oriundos da sincronização foi de R $\$ 452,40 /$ bezerro e, multiplicando-se pelo número de bezerros existentes em cada grupo, obtiveram-se R \$29.406,00; R \$26.239,20; R \$ 14.929,20; e R\$ $16.738,80$, para o GPLP, GPCP, GPPLP e GPPCP, respectivamente. No acasalamento aos 26 meses no GPLP, foram obtidos 83,5 bezerros; no GPCP 84,2 bezerros; no GPPLP 86,7 bezerros; no GPPCP 86,3 bezerros e no GCF 90 bezerros. Com a venda dos bezerros, os GPLP, GPCP, GPPLP e GPPCP e GCF geraram, respectivamente, R $\$ 37.775,40$; $\mathrm{R} \$ 38.092,08 ; \mathrm{R} \$ 39.223,08 ; \mathrm{R} \$ 39.042,12$ e $\mathrm{R} \$ 40.716,00$.
Como observado na análise estatística dos resultados biológicos, houve diferença na taxa de prenhez apenas entre as fêmeas púberes e pré-púberes. Portanto, foram desconsiderados os tratamentos de exposição prolongada à progesterona na comparação dos resultados econômicos. Desta forma, houve diferença nas receitas obtidas entre as fêmeas púberes, pré-púberes e acasaladas somente aos 26 meses (GCF). Ao considerar as fêmeas púberes como base fixa, verificou-se que esses animais propiciaram retorno de 12 e 35\% maior que o das pré-púberes e o das GCF, respectivamente. Quando utilizadas como base fixa em relação ao GCF, as fêmeas pré-púberes tiveram receita $26 \%$ superior, informação confirmadas na análise dos indicadores financeiros (Tabela 2), uma vez que a maior taxa interna de retorno (TIR) e valor presente líquido (VPL) foi observada para GPLP e GPCP. Outras vantagens não consideradas neste trabalho foram a diluição dos custos fixos, o maior giro de capital e a redução do estoque improdutivo da propriedade em comparação ao sistema de acasalamento aos 26 meses.

Tabela 2 - Receitas totais das venda dos bezerros dos diferentes grupos, em R\$, e receita obtida da venda de bezerros oriundos do acasalamento aos 16 meses

\begin{tabular}{|c|c|c|c|c|c|}
\hline Item & GPLP & GPCP & GPPLP & GPPCP & GCF \\
\hline Valor em R\$ & $29.406,00$ & $26.239,20$ & $14.929,20$ & $16.730,00$ & - \\
\hline \multicolumn{6}{|l|}{$\begin{array}{l}\text { Receita obtida da venda de bezerros oriundos } \\
\text { do acasalamento aos } 26 \text { meses }\end{array}$} \\
\hline Valor em R\$ & $37.775,40$ & $38.092,08$ & $39.223,08$ & $39.042,12$ & $40.716,00$ \\
\hline Receita total, R\$ & $67.181,40$ & $64.331,28$ & $54.152,28$ & $55.772,12$ & $40.716,00$ \\
\hline \multicolumn{6}{|l|}{$\begin{array}{l}\text { Indicadores financeiros dos sistemas de } \\
\text { produção analisados }\end{array}$} \\
\hline Taxa interna de retorno, $\%$ ao ano & 7,57 & 1,75 & 1,39 & 2,98 & $-8,33$ \\
\hline Taxa mínima de atratividade, \% ao mês & 0,85 & 0,85 & 0,85 & 0,85 & 0,85 \\
\hline Valor presente líquido, em R $\$-36$ meses & $-378,43$ & $-2.337,06$ & $-2.273,04$ & $-1.849,79$ & $-11.769,36$ \\
\hline
\end{tabular}




\section{Conclusões}

A exposição prolongada a um progestágeno não é eficiente para aumentar a taxa de prenhez de novilhas prépúberes e púberes submetidas à inseminação artificial em tempo fixo, no entanto, a taxa de prenhez é maior nas novilhas púberes. Considerando a diferença na taxa de prenhez entre novilhas púberes e pré-púberes, há vantagens econômicas na utilização de protocolos com progestágeno em novilhas de corte aos 16 meses em comparação a resultados simulados de um sistema tradicional de acasalamento aos 26 meses.

\section{Literatura Citada}

ANDERSON, L.H.; MCDOWELL, C.M.; DAY, M.L. Progestininduced puberty and secretion of luteinizing hormone in heifers. Biology of Reproduction, v.54, p.1025-1031, 1996.

BUARQUE, C. Avaliação econômica de projetos. 21.ed. Rio de Janeiro: Editora Campus, 1984. 266p.

CASTILHO, E.M.; PFEIFER, L.F.M. Influência do momento da aplicação de prostaglandina em protocolos utilizando progestágenos em vacas de corte pós-parto. A Hora Veterinária, v.26, n. 152, p. $17-23,2005$.

DAY, M.L.; IMAKAWA, K.; GARCIA-WINDER, M. et al. Endocrine mechanisms of puberty in heifers. Estradiol negative feedback regulation of luteinizing hormone secretion. Biology of Reproduction, v.31, p.332-341, 1984.

DONALDSON, L.E.; BASSETT, J.M.; THORBURN, G.D. Peripheral plasma progesterone concentration of cows during puberty, oestrous cycles, pregnancy, and lactation, and the effect of undernutrition or exogenous oxytocin on progesterone concentration. Journal of Endocrinology, v.48, p.599-614, 1970.

GONZALEZ-PADILLA, E.; NISWENDER, G.D.; WILTBANK, J.N. Puberty in beef heifers. II. Effect of injections of progesterone and estradiol-17b on serum LH, FSH and ovarian activity. Journal of Animal Science, v.40, p.1105-1109, 1975a.

GONZALEZ-PADILLA, E.; WILTBANK, J.N.; NISWENDER, G.D. Puberty in beef heifers. I. The interrelationship between pituitary, hypothalamic, and ovarian hormones. Journal of Animal Science, v.40, p.1091-1104, 1975b.

GONZALEZ-PADILLA, E.; RUIZ, R.; LEFEVER, D. et al. Puberty in beef heifers. III. Induction of fertile estrus. Journal of Animal Science, v.40, p.1110-1118, 1975c.

GREGORY, R.M.; ROCHA, D.C. Protocolos de sincronização e indução de estros em vacas de corte no Rio Grande do Sul. In: SIMPÓSIO INTERNACIONAL DE REPRODUÇÃO ANIMAL APLICADA, 1., 2004, Londrina. Anais... São Paulo: Faculdade de Medicina Veterinária e Zootecnia, Universidade de São Paulo. 2004. p.147-154.

HALL, J.B.; STAIGMILLER, R.B.; SHORT, R.E. et al. Effect of age and pattern of gain on induction of puberty with a progestin in beef heifers. Journal of Animal Science, v.75, p.1606-1611, 1997.
IMWALLE, D.B.; PATTERSON, D.J.; SCHILLO, K.K. Effects of melengestrol acetate on onset of puberty, follicular growth, and patterns of luteinizing hormone secretion in beef heifers. Biology of Reproduction, v.58, p.1432-1436, 1998.

LAMB, G.C.; LARSON, J.E.; GEARY, T.W. et al. Synchronization of estrus and artificial insemination in replacement beef heifers using gonadotropin-releasing hormone, prostaglandin F $2 \alpha$, and progesterone. Journal of Animal Science, v.84, p.3000-3009, 2006.

LESMEISTER, J.L.; BURFENING, P.J.; BLACKWELL, R.L. Date of first calving in beef cows and subsequent calf production. Journal of Animal Science, v.36, p.1, 1973.

LOBATO, J.F.P.; AZAMBUJA, P.S. Recria de terneiras e eficiência reprodutiva de novilhas e vacas primíparas. In: BORGES, J.B.S.; GREGORY, R.M. SIMPÓSIO DE REPRODUÇÃO BOVINA - SINCRONIZAÇÃO DE ESTROS EM BOVINOS, 2002, Porto Alegre. Anais... Porto Alegre: Gráfica Jacuí, 2002. p. 5-17.

LOBATO, J.F.P.; DERESZ, F.; LEBOUTE, E.M.; et al. Pastagens melhoradas e suplementação alimentar no comportamento reprodutivo de vacas de corte primíparas. Revista Brasileira de Zootecnia, v.27, n.1, p.47-53, 1998a.

LOBATO, J.E.P.; ZANOTTA, R.L.D.; PEREIRA, O.A.N. Efeitos das dietas pré e pós-parto de vacas primíparas sobre o desenvolvimento dos bezerros. Revista Brasileira de Zootecnia, v.27, n.5, p.863-867, 1998b.

LOPES, M.A.; CARVALHO, F.M. Gestão na pecuária de corte: custo de produção e análise de rentabilidade. In: SIMPÓSIO PFIZER SOBRE REPRODUÇÃO, DOENÇAS INFECCIOSAS E VACINAS DE BOVINOS, 6., 2003, Porto Alegre. Anais... São Paulo: Laboratórios Pfizer, 2003. p.33-46.

LUCY, M.C.; BILLINGS, H.J.; BUTLER, W.R. et al. Efficacy of an intravaginal progesterone insert and an injection of PGF $2 \alpha$ for synchronizing estrus and shortening the interval to pregnancy in postpartum beef cows, peripubertal beef heifers, and dairy heifers. Journal of Animal Science, v.79, p.982-995, 2001.

RASBY, R.J.; DAY, M.L.; JOHNSON, S.K. et al. Luteal function and estrus in peripubertal beef heifers treated with an intravaginal progesterone releasing device with or without a subsequent injection of estradiol. Theriogenology, v.50, p.55-63, 1998.

SILVA, M.D.; BARCELlOS, J.O.J.; PRATES, E.R. Desempenho reprodutivo de novilhas de corte acasaladas aos 18 ou aos 24 meses de idade. Revista Brasileira de Zootecnia, v.34, n.6, p.2057-2063, 2005.

STATISTIX $^{\circledR}$. Statistix for Widows user's manual. Tallahassee: Analytical software, 2004. (CD-ROM).

TANAKA, Y.; VINCENT', D.L.; LEDGERWOOD, K.S. et al. Variable progesterone response and estradiol secretion in prepubertal beef heifers following treatment with norgestomet implants. Theriogenology, v.43, p.1077-1086, 1995.

TAUCK, S.A.; WILKINSON, J.R.C.; OLSEN, J.R. et al. Comparison of controlled internal drug release device and melengesterol acetate as progestin sources in an estrous synchronization protocol for beef heifers. Theriogenology, v. 68, p. $162-167,2007$

VIEIRA, A.; LOBATO, J.F.P.; CORRÊA, E.S. et al. Desenvolvimento e desempenho reprodutivo de novilhas Nelore criadas a pasto nos cerrados do Centro-Oeste brasileiro. Revista Brasileira de Zootecnia, v.35, n.1, p.186-192, 2006. 